\title{
0479 INTANGIBLE COST OF ROAD TRAFFIC INJURIES: A PHENOMENOLOGICAL APPROACH
}

R Pérez-Nunez*, B Pelcastre-Villafuerte, M Hijar-Medina, A Vila-Burgos, A Celis Correspondence: National Institute of Public Health (Mexico), Universidad No. 655 Col. Santa Mara Ahuacatitln Cerrada los Pinos y Caminera. Cuernavaca Morelos 62100, Mexico

10.1136/ip.2010.029215.479

To explore the consequences of road traffic injury (RTI) in terms of health, individual-family life and its implications on the households economies, the experience of 12 injured and 12 relatives of RTI-deaths was documented using phenomenological approach. Semistructured interviews were analysed throughout critical discourse analysis. Changes in physical and mental health were observed in both injured and relatives of those who died from RTI what implied changes in daily activities temporally or permanently and even to the redefinition of future life. People also change the way they see and act in life learning from their experience: preventive attitudes were evident in their discourse. This also represents a teaching experience for others: consciously and unconsciously survivors transmit a road safety message. Changes in family life were as simple as acquisition of new responsibilities or roles inside the household and as complicated as changes in families composition, sometimes affecting intra-familial relationships and translating in family tension and problems, although occasionally makes families become closer. Associated unexpected expenditures and loss of income have consequences in the short, medium and long term that unbalance households economies and immerse people into a constant stress. Individuals and families future plans are occasionally conditioned to whether they have or not debts. Economic dependency was sometimes observed as well as uncertainty about future life (ie, households sustainability). Sometimes, households change and adapt their life to what they now are able to afford, having important repercussions. Consequences observed in the individuals discourse have no sense in monetary terms. Evidencing what people live after a RTI, allows us to learn from their experience and highlights the need of preventing these events. 